\title{
Characterizing metabolic interactions in a clostridial co-culture for consolidated bioprocessing
}

Fahimeh Salimi and Radhakrishnan Mahadevan *

\begin{abstract}
Background: Clostridial co-culture containing cellulolytic and solventogenic species is a potential consolidated bioprocessing (CBP) approach for producing biochemicals and biofuels from cellulosic biomass. It has been demonstrated that the rate of cellulose utilization in the co-culture of Clostridium acetobutylicum and Clostridium cellulolyticum is improved compared to the mono-culture of C. cellulolyticum (BL 5:119-124, 1983). However, the metabolic interactions in this co-culture are not well understood. To investigate the metabolic interactions in this co-culture we dynamically characterized the physiology and microbial composition using qPCR.

Results: The GPCR data suggested a higher growth rate of $C$. cellulolyticum in the co-culture compared to its mono-culture. Our results also showed that in contrast to the mono-culture of $C$. cellulolyticum, which did not show any cellulolytic activity under conditions similar to those of co-culture, the co-culture did show cellulolytic activity even superior to the C. cellulolyticum mono-culture at its optimal pH of 7.2. Moreover, experiments indicated that the co-culture cellulolytic activity depends on the concentration of $C$. acetobutylicum in the co-culture, as no cellulolytic activity was observed at low concentration of $C$. acetobutylicum, and thus confirming the essential role of $C$. acetobutylicum in improving C. cellulolyticum growth in the co-culture. Furthermore, butanol concentration of $350 \mathrm{mg} / \mathrm{L}$ was detected in the co-culture batch experiments.

Conclusion: These results suggest the presence of synergism between these two species, while C. acetobutylicum metabolic activity significantly improves the cellulolytic activity in the co-culture, and allows C. cellulolyticum to survive under harsh co-culture conditions, which do not allow C. cellulolyticum to grow and metabolize cellulose independently. It is likely that $C$. acetobutylicum improves the cellulolytic activity of $C$. cellulolyticum in the co-culture through exchange of metabolites such as pyruvate, enabling it to grow and metabolize cellulose under harsh co-culture conditions.
\end{abstract}

Keywords: Consolidated bioprocessing, Clostridial co-culture, qPCR analysis, Clostridium acetobutylicum, Clostridium cellulolyticum

\section{Background}

In consolidated bioprocessing (CBP) all four biological steps involved in the conversion of cellulosic biomass, which includes production of saccharolytic enzymes, hydrolysis of the polysaccharides present in pre-treated biomass, and fermentation of hexose and pentose sugars present in the hydrolyzate to desired products, takes place in one bioreactor using a single microorganism or a microbial consortium without the external addition of saccharolytic enzymes [1]. Consolidated bioprocessing has been proposed to decrease the production cost by

\footnotetext{
* Correspondence: krishna.mahadevan@utoronto.ca

Department of Chemical Engineering and Applied Chemistry, University of Toronto, 200 College Street, Toronto, ON M5S 2E6, Canada
}

eliminating the costs associated with the cellulase production stage. CBP requires microorganisms with both rapid conversion of cellulose and high product yield, productivities and titres, while such microbes have not been identified in nature yet and need to be developed [2-5]. To realize this aim, two strategies have be applied: the native strategy which improves the product formation capabilities, such as yield and titre in natural cellulolytic microorganisms [6-11], and the recombinant strategy that involves engineering non-cellulolytic organisms with high product yields so that they will express heterologous cellulase and be able to utilize cellulose $[1,5,12]$.

An alternative method for the production of biobutanol and biochemicals from cellulosic biomass in a consolidated

\section{Biomed Central}


bioprocessing approach is the use of mesophilic clostridial co-culture. Clostridium acetobutylicum shows an effective capability to ferment cellulose derived sugars as well as hemicellulose derived sugars, such as cellobiose, mannose, arabinose, xylose, glucose, and galactose to acetone, butanol, and ethanol [13-16]. Thus, the co-culture of this bacterial species with a mesophilic cellulose degrading bacterium can be an efficient approach. Clostridium cellulolyticum is a cellulolytic, mesophilic bacterium which is able to solublize crystalline cellulose in pretreated hardwood [17]. A simplified scheme of this clostridial co-culture fermentation on cellulose is presented in Figure 1. C. cellulolyticum synthesizes cellulosome, which is an extracellular multi-enzymatic complex, and degrades cellulose, with the use of this cellulosome, to glucose and soluble cellodextrins (mainly cellobiose), which can be fermented by both species in the co-culture.

The co-culture of C. cellulolyticum with C. acetobutylicum has been studied previously, and it has been shown that cellulolytic activity is the limiting factor in the coculture fermentation since most of the cellulase activity products are consumed by $C$. acetobutylicum. The fermentation products have been mainly butyric acid along with butanol, acetic acid and ethanol, and the lack of glucose, which is required for solvent production due to low cellulolytic activity, was hypothesized to be the reason for acid accumulation [18,19]. Furthermore, three times more cellulosic material was degraded in the co-culture compared to the mono-culture of $C$. cellulolyticum due to the utilization of cellulase activity products and the elimination of their repressive effects [20], suggesting

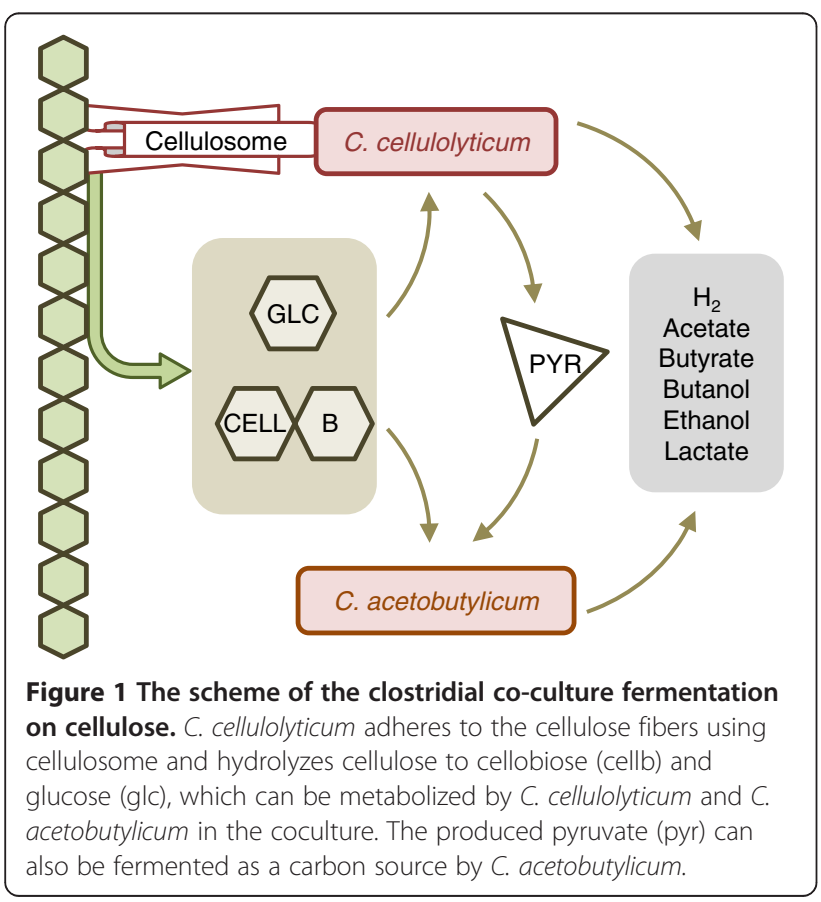

the presence of synergism between these two species. The analysis of this effect can be valuable for optimizing the rate of cellulosic material degradation.

Therefore, in this study, we investigated metabolic interactions in this co-culture by developing a comparative qPCR analysis of the co-culture and mono-cultures of C. cellulolyticum and C. acetobutylicum. Investigation of the metabolism in this clostridial co-culture along with the mono-cultures revealed that significant increase in the rate of cellulose hydrolysis can be achieved using the co-culture and making use of the synergism existing between the two clostridial species. It is likely that $C$. acetobutylicum improves the cellulolytic activity of $C$. cellulolyticum in the co-culture through exchange of metabolites such as pyruvate, enabling it to grow and metabolize cellulose under harsh co-culture conditions. This clostridial co-culture can offer a considerable potential CBP approach for producing commodity chemicals from cellulosic biomass, taking advantage of C. acetobutylicum metabolic potential in converting sugars to variety of chemicals.

\section{Results and discussion}

\section{Experimental characterization of $C$. cellulolyticum cellulolytic activity at high cellulose concentration, addressing the $\mathrm{pH}$ effect}

In the previous co-culture study [19], the $\mathrm{pH}$ effect on C. cellulolyticum growth and cellulolytic activity has not been addressed; therefore, to investigate the $\mathrm{pH}$ effect on $C$. cellulolyticum growth and cellulolytic activity in a mono-culture at high cellulose concentration of $20 \mathrm{~g} / \mathrm{L}$, two batch cultures of $C$. cellulolyticum were conducted: one batch at $\mathrm{pH}$ of 7.2, which is an optimal $\mathrm{pH}$ for C. cellulolyticum growth and cellulolytic activity [21], and another batch with the same $\mathrm{pH}$ profile as the co-culture run, i.e. initially at $\mathrm{pH}$ of 7.0 for 2 days followed by a $\mathrm{pH}$ switch to 6.0. The profiles of cellulose solubilization and biomass concentration are shown in Figure 2. In the mono-culture at $\mathrm{pH}$ of 7.2, TOC data showed that $1.5 \mathrm{~g} / \mathrm{L}$ of carbon was solubilized after 14 days, which is equivalent to $4.9 \mathrm{~g} / \mathrm{L}$ of cellulose degraded, assuming $24 \%$ of carbon flow goes towards $\mathrm{CO}_{2}$ formation; this amount of degraded cellulose was comparable to $5.1 \mathrm{~g} / \mathrm{L}$ reported for the C. cellulolyticum mono-culture at the same initial cellulose concentration $[19,22]$. Also, qPCR data showed that cells reached a stationary growth phase after 9 days with a maximum cell density of $5 \times 10^{9} \mathrm{cell} / \mathrm{mL}$ and a specific growth rate of 1.23 day $^{-1}$.

In contrast, the mono-culture run under co-culture $\mathrm{pH}$ profile did not show any cellulose solubilization during the batch (Figure 2), and cells had a very low growth rate of 0.16 day $^{-1}$ (87\% decrease) compared to the monoculture batch at $\mathrm{pH}$ of 7.2. The effect of $\mathrm{pH}$ on C. cellulolyticum growth and cellulolytic activity at low cellulose 


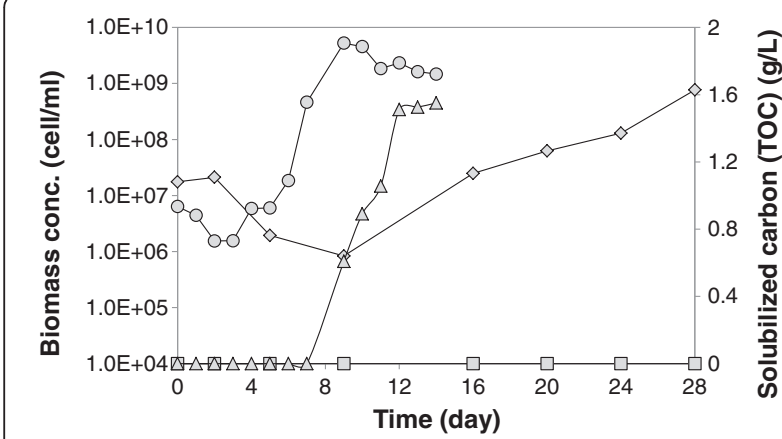

Figure 2 The total solubilized carbon and biomass profiles in the $C$. cellulolyticum mono-culture experiments. The biomass profile in the mono-culture at $\mathrm{pH}$ of 7.2 (circle), TOC profile in the mono-culture at $\mathrm{pH}$ of 7.2 (triangle), biomass profile in the mono-culture under $\mathrm{pH}$ profile (rhombus), and TOC profile in the mono-culture under $\mathrm{pH}$ profile (square).

concentration of $3.7 \mathrm{~g} / \mathrm{L}$ has been addressed previously [21], and it has been shown that C. cellulolyticum is significantly affected by acidic $\mathrm{pH}$, where a $\mathrm{pH}$ drop from 7.0 to 6.4 leads to fourfold lower biomass concentration. It has been suggested that acidic $\mathrm{pH}$ hampers biomass formation, likely through direct effect of $\mathrm{pH}$ on a cellular constituent such as an enzyme or a transport protein, rather than cellulose degradation capability in C. cellulolyticum. This argument is supported by the observation that the flux through cellulose degradation reaction remains almost unvarying, in the range of 1.69 to $1.84 \mathrm{mmol}$ (g cell. $\mathrm{h})^{-1}$, while $\mathrm{pH}$ varies between 6.4 to 7.0 [21]. However, regardless of the mechanism of inhibition, this $\mathrm{pH}$ effect must be considered when comparing cellulolytic activity and C. cellulolyticum growth and metabolism in the mono-culture and co-culture.

\section{Experimental characterization of the co-culture metabolism}

To prepare the co-culture medium for the first co-culture experiment $\mathrm{A}$, all media components including the iron solution were autoclaved. The profile of cellulose degradation in this co-culture is shown in Figure 3a. At initial cellulose concentration of $20 \mathrm{~g} / \mathrm{L}$, which is equivalent to $6.08 \mathrm{~g} / \mathrm{L}$ of final TOC concentration, assuming full degradation of cellulose and considering $24 \%$ of the total carbon would be used toward $\mathrm{CO}_{2}$ formation, about $82 \%$ of cellulose was degraded after 28 days in the co-culture experiment A. Compared to the mono-culture at optimal $\mathrm{pH}$ of 7.2, cellulose degradation showed about $82 \%$ improvement as shown in Figure 3c, whereas no cellulose degradation was observed in the mono-culture run under co-culture $\mathrm{pH}$ profile. These results confirm that $C$. acetobutylicum metabolic activity significantly improves the cellulolytic activity in the co-culture, and in fact makes it possible for C. cellulolyticum to survive

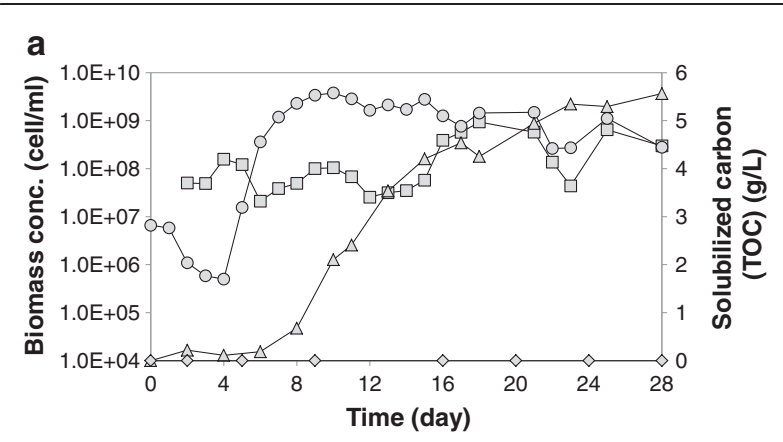

b
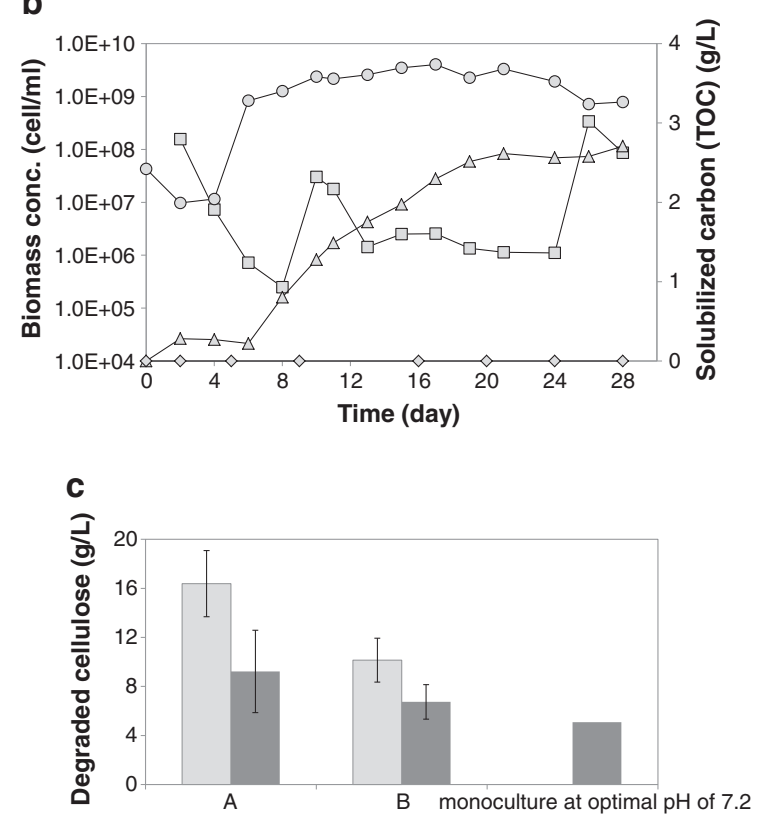

Figure 3 The cellulose solubilization in the co-culture and mono-culture experiments. (a) The profiles of cellulose

degradation and biomass formations in the co-culture experiment $\mathrm{A}$ (with autoclaved iron solution), where figure (a) shows one data set representative of two independent experiments, with both showing comparable results; (b) The profiles of cellulose degradation and biomass formations in the co-culture experiments B (with filter sterilized iron solution), where figure (b) shows one data set representative of two independent experiments, with both showing comparable results. The time profiles of C. cellulolyticum (circle), C. acetobutylicum (square) and cellulose solubilization in terms of total organic carbon in each co-culture (triangle) at initial cellulose concentration of $20 \mathrm{~g} / \mathrm{L}$ and under $\mathrm{pH}$ profile, along with cellulose solubilization in terms of total organic carbon in C. cellulolyticum mono-culture under the same co-culture conditions (rhombus). C. acetobutylicum was inoculated after 2 days; (c) Degradation of cellulose in the co-culture experiments $\mathrm{A}$ and $\mathrm{B}$ and the mono-culture experiment at optimal pH of 7.2, 5 days after reaching the maximum C. cellulolyticum concentration in the culture (dark grey) and after 28 days (light grey). Error bars are based on two duplicate experiments.

under harsh co-culture conditions, which do not allow it to grow and metabolize cellulose independently.

Furthermore, to check and ensure iron sufficiency in the co-culture medium, the co-culture medium was prepared 
by filter sterilizing the ferrous sulphate solution (experiment $\mathrm{B}$ ), rather than autoclaving as for co-culture experiment $\mathrm{A}$, to avoid potential iron oxidation during medium preparation. The results of this experiment are shown in Figure 3b; while the cellulolytic activity was high in the co-culture experiment $\mathrm{B}$, and $C$. cellulolyticum growth rate was still as high as experiment $\mathrm{A}$, but the cellulose degradation was declined by $38 \%$ compared to experiment A, as it is presented in Figure 3c. Cellulose degradation in each batch was estimated from carbon balance and TOC measurements, taking into account that about $24 \%$ of the total carbon has been utilized for $\mathrm{CO}_{2}$ formation, as described in analysis section. These results confirmed that the co-culture was not under iron limiting condition, and the presence of more ferrous ions had an adverse effect on the co-culture cellulolytic activity.

The mechanism underlying such a synergy between the two clostridial species is not clearly understood. Hence, in order to understand the nature of interactions between C. acetobutylicum and C. cellulolyticum, that improve cellulose solubilization, we used qPCR to track the population of each species in the batch cultures. Figure $3 \mathrm{a}$ and b show the dynamic profiles of each species population in the co-culture batches. C. cellulolyticum biomass concentration reached to $3 \times 10^{9} \mathrm{cell} / \mathrm{mL}$ in the co-cultures, which was the same value for maximum biomass concentration in the mono-culture runs; however the growth dynamics was significantly faster in the co-cultures compared to the mono-culture run under the same $\mathrm{pH}$ profile. C. cellulolyticum growth rate in the co-culture was comparable to its growth rate in mono-culture under optimal $\mathrm{pH}$ condition of 7.2. Also, it could be observed from all co-culture TOC profiles that cellulose solubilization started after C. cellulolyticum had reached its late exponential growth phase.

C. acetobutylicum biomass profiles are also shown in Figure 3, where the initial decrease in biomass concentration in the co-culture could be attributed to the lack of available sugars for $C$. acetobutylicum to grow while C. cellulolyticum had been in the lag phase. Furthermore, a considerable growth could be observed for $C$. acetobutylicum, when $C$. cellulolyticum had reached its maximum concentration in the co-culture, where possibly more sugars became available in the co-culture to support C. acetobutylicum growth. Although C. acetobutylicum biomass concentration showed significant increases at some points over the course of co-culture, it was fluctuating and did not remain constant. It has been suggested that in the microbial communities growing on cellulosic material, where there is a competition between cellulose-adherent cellulolytic microorganisms and non-adhered microbes for cellulose hydrolysis products, cellulose-adherent cellulolytic microorganisms are possibly successful winners [2], and this phenomenon could explain the limited growth of C. acetobutylicum in the co-culture.
In addition, $C$. acetobutylicum has cellobiase and endoglucanase activities, but is not able to hydrolyze crystalline cellulose due to lack of the required enzymatic activities [23], although it produces cellulosome [24]; therefore, the improved cellulolytic activity in the co-culture cannot be attributed to $C$. acetobutylicum cellulolytic activity. However, C. acetobutylicum is able to ferment cellobiose and cellulose-derived sugars, and the improved cellulolytic activity in co-culture can be attributed to the role of $C$. acetobutylicum in consuming sugars and preventing the carbon overflow toward C. cellulolyticum, as C. cellulolyticum is unable to metabolize high concentrations of cellobiose [25].

Also, degree of synergism (DS), defined as the activity of a mixture of components divided by the sum of the component activities evaluated separately [26], in this co-culture can be estimated as the ratio of C. cellulolyticum growth rates in co-culture and mono-culture under $\mathrm{pH}$ profile, and was equal to 7.99; although the co-culture DS determined based on the cellulolytic activities in the co-culture and the mono-culture would be substantially high, which indicates the presence of a strong synergism in this clostridial co-culture. The DS value of 5 or higher is not very common in enzyme-microbe cellulose hydrolysis systems and has been observed under some conditions $[27,28]$. Moreover, in this co-culture on fibrous cellulose, observed maximum cellulose degradation rate of 0.108 $\mathrm{g} /(\mathrm{L} . \mathrm{h})$ is comparable with cellulose degradation rate of $0.15 \mathrm{~g} /(\mathrm{L} . \mathrm{h})$ in C. thermocellum culture on crystalline cellulose, which shows one of the highest cellulose utilization rates among cellulolytic microorganisms [26].

Since cellulolytic bacteria are unable to grow at low intracellular $\mathrm{pH}$, under acidic environment the $\mathrm{pH}$ gradient $(\Delta \mathrm{pH})$ across the cell membrane is high; consequently, the intracellular dissociation of fermentation acids, which are membrane permeable in undissociated form, and the intracellular accumulation of acid anions lead to anion toxicity, which is the likely reason of growth inhibition under acidic condition [29,30]. Furthermore, it has been shown that presence of lactate and acetate ions in an acidic medium leads to a significant decline of glutamate synthesis in Clostridium sporogenes MD1, which inhibits the bacterial growth [30]. Also, for E. coli culture at $\mathrm{pH}$ of 6 , incubation of cells with $8 \mathrm{mM}$ acetate for $20 \mathrm{~min}$ was shown to result in intracellular accumulation of acetate anions $(240 \mathrm{mM})$, and reduced level of intracellular glutamate pools [31]. Furthermore, in mildly acidic E. coli cultures (pH of 6), inhibition of methionine biosynthesis by acetate $(8 \mathrm{mM})$ and the toxicity of accumulated homocysteine have been indicated as the cause of growth inhibition by acetate under weak acid stress [32]. Addition of methionine to this culture can restore $E$. coli growth rate to some significant extent. This effect has been also reported for other organic acids. 
In this clostridial co-culture, the synergy could be attributed to the exchange of some growth precursors and biomass constituents between $C$. acetobutylicum and C. cellulolyticum, which potentially enables the cellulolytic organism to grow and metabolize cellulose under acidic $\mathrm{pH}$ condition. C. acetobutylicum is a fermentative bacterium which is able to grow well under acidic conditions in acidogenic and solventogenic growth phases. The results of co-culture experiment under low concentration of $C$. acetobutylicum, which are presented in the next section, also provides support for the role of C. acetobutylicum.

\section{Experimental characterization of the co-culture metabolism} with low concentration of $C$. acetobutylicum

To investigate if high initial concentration of C. acetobutylicum contributes to its low growth in the co-culture, and if $C$. acetobutylicum growth in the co-culture is affected by the ratio of cells to the cellulose hydrolysate, a coculture experiment was conducted with 100 times lower initial concentration of $C$. acetobutylicum. $2 \mathrm{~mL}$ of C. acetobutylicum culture at exponential growth phase was centrifuged and the cell pellets were suspended in 2 $\mathrm{mL}$ of CGM medium and incoculated into the bioreactor. The results of this experiment are presented in Figure 4, where no cellulose degradation was observed in either biological replicates. Also, C. cellulolyticum as well as $C$. acetobutylicum did not grow in the co-culture. This experiment confirmed that the role of $C$. acetobutylicum in the metabolism of cellulose by the co-culture is associated with the population of $C$. acetobutylicum, and can be attributed to the exchange of some metabolites between the two species.

Furthermore, the metabolic behavior of C. acetobutylicum under the co-culture conditions was investigated

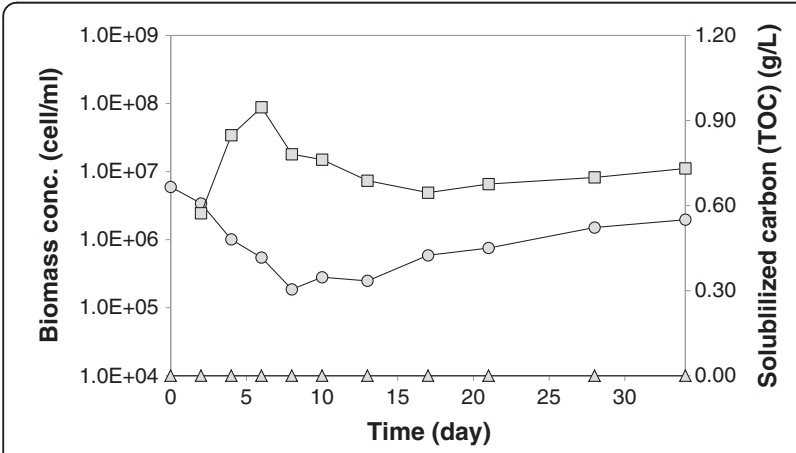

Figure 4 The profiles of cellulose degradation and biomass formations in the co-culture experiments with low concentration of $\boldsymbol{C}$. acetobutylicum. The time profiles of C. cellulolyticum (circle), C. acetobutylicum (square) and cellulose solubilization in terms of total organic carbon (triangle) in the co-culture experiment with C. acetobutylicum inoculated after 2 days. The figure shows one data set representative of two independent experiments, with both showing comparable results.
(Additional file 1), where this study confirmed the metabolism of pyruvate and the released sugars by $C$. acetobutylicum in the clostridial co-culture, and that the observed oscillations in the $C$. acetobutylicum concentration in the co-cultures could be due to the slow release of sugars by $C$. cellulolyticum that can lead to starvation cycles for C. acetobutylicum in the co-culture.

\section{Analysis of product formations in the co-culture}

Figure 5 shows the ranges for co-culture and monoculture product concentrations after 28 days. As it can be noted, acetate, ethanol, lactate, butyrate, and butanol were the main products of the fermentation in the co-culture. Butyrate appeared after $C$. acetobutylicum inoculation in the co-culture, but its concentration remained low. Neither acetate nor butyrate uptake, which are the characteristics of the solventogenic phase in C. acetobutylicum metabolism, was observed in this co-culture. At high cellulose concentration, $C$. cellulolyticum produces lactate as its main product along with acetate and ethanol [22]. The lactate uptake, observed in the co-culture batches, coincided with butanol formation (Additional file 1). The lactate uptake can be related to C. acetobutylicum metabolic activity, as $C$. acetobutylicum ATCC 824 has been shown to co-ferment lactate and glucose [33].

It has been previously shown [22] that in $\mathrm{pH}$ controlled batch cultures of $C$. cellulolyticum on a defined medium, the distribution of carbon flow depends on the initial cellulose concentration. For concentrations less than $6.7 \mathrm{~g} / \mathrm{L}$ of cellulose, acetate, ethanol, $\mathrm{CO}_{2}$ and $\mathrm{H}_{2}$ were shown to be the main fermentation end products and more than $91 \%$ of cellulose was observed to be degraded. At higher cellulose concentrations, from $6.7 \mathrm{~g} / \mathrm{L}$ up to 29.1 $\mathrm{g} / \mathrm{L}$, carbon flow is redirected from ethanol and acetate towards lactate and extracellular pyruvate. In addition, in batch cultures of $C$. cellulolyticum on high cellulose concentration, it has been shown that the peak of

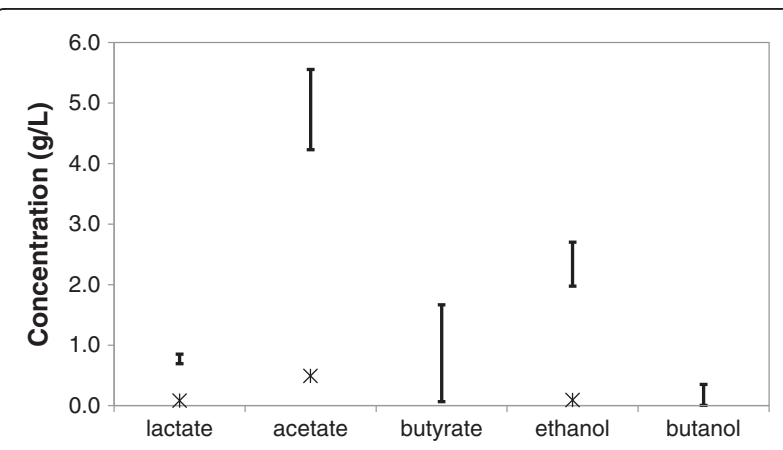

Figure 5 Product concentrations in the co-cultures and the mono-culture under $\mathrm{pH}$ profile. Product concentration ranges in the co-culture experiment A after 28 days (ranges have been calculated based on two sets of experimental data), and the product concentrations in the mono-culture batch under $\mathrm{pH}$ profile after 28 days (asterisk). 
pyruvate formation coincides with the start of lactate formation, and this pyruvate accumulation in the C. cellulolyticum culture shows that the rate of cellulose catabolism is higher than the rate of pyruvate consumption. Also it has been suggested that the cellulose hydrolysis depends on the concentration of $C$. cellulolyticum, which remains constant at and above $6.7 \mathrm{~g} / \mathrm{L}$ of cellulose [22].

Furthermore, it has been shown that re-inoculating a fresh culture of $C$. cellulolyticum at high cellulose concentration of $29.1 \mathrm{~g} / \mathrm{L}$, where substrate is not fully consumed, significantly improves the cellulose solubilization and biomass yield compared to a classical batch [22]. This result indicates that the incomplete cellulose catabolism is not due to either the limitation of adhesion sites on cellulose fibers or product inhibition. At high cellulose concentrations, the likely explanation for the incomplete cellulose consumption is the lack of control on carbon uptake flow and an imbalanced metabolism leading to the accumulation of intracellular metabolites and selfintoxication of the cells, eventually resulting in a growth arrest [22,34]. Similarly, extracellular pyruvate formation has been reported in $C$. thermocellum cultures at high cellulose and cellobiose concentrations, which evidences the overflow metabolism [35].

In our experiments, the maximum concentration of $C$. cellulolyticum in co-culture experiments was the same as the mono-culture experiment under optimal $\mathrm{pH}$ of 7.2 , however the cellulose degradation was improved up to $82 \%$ (Figure 3c), which confirms the role of C. acetobutylicum in cellulose degradation, while $C$. cellulolyticum has reached the stationary growth phase. We observed pyruvate accumulation of $0.029 \mathrm{~g} / \mathrm{L}$ in the mono-culture batch under the co-culture $\mathrm{pH}$ profile and $0.004 \mathrm{~g} / \mathrm{L}$ in the mono-culture batch at $\mathrm{pH}$ of 7.2. In the co-culture replicates, maximum pyruvate concentration of $0.17 \mathrm{~g} / \mathrm{L}$ was observed, which was taken up later during the course of experiments coinciding with butyrate formation in the co-cultures. Our previous modeling studies have suggested that limited pyruvate-ferredoxin oxidoreductase (PFO) activity, which cannot support high pyruvate flow, results in pyruvate overflow [36]. Hence, a potential explanation for pyruvate secretion in C. cellulolyticum cultures is the limitation on the pyruvate consumption rate and a comparatively higher carbon catabolism rate, and due to inefficient regulation of entering carbon flow [25]. Furthermore, intracellular pyruvate accumulation could be the explanation for the growth arrest at high cellulose concentrations [37], at which cells switch to stationary growth phase before substrate depletion.

Pyruvate uptake in the co-culture can be explained by the capability of $C$. acetobutylicum to metabolize pyruvate. It has been also reported that providing $C$. acetobutylicum with pyruvate as the sole carbon source results in the growth and production of mainly acetate and butyrate
[38]. In another co-culture study, the removal of C. cellulolyticum metabolic products such as pyruvate and their associated inhibitory effects, by Rhodopseudomonas palustris in the co-culture of C. cellulolyticum and R. palustris has been reported as the underlying reason for the improved cellulose degradation and bacterial growth in this co-culture [39]. C. cellulolyticum growth on cellulose has been shown to be severely inhibited by pyruvate; where about $60 \%$ decrease in the biomass concentration in the presence of $2 \mathrm{mM}(176 \mathrm{mg} / \mathrm{L})$ pyruvate has been observed in C. cellulolyticum mono-culture [39]. Therefore, pyruvate removal by $C$. acetobutylicum and alleviating its inhibitory effect can be a contributing factor in the improved growth of $C$. cellulolyticum and its boosted cellulolytic activity in the co-culture.

The major products of pyruvate fermentation by $C$. acetobutylicum are acetate, butyrate and butanol, and neither acetate nor butyrate is reutilized. The effects of pyruvate on glucose fermentation by $C$. acetobutylicum have also been investigated before, and it has been shown that both substrates can be fermented simultaneously [40]. Furthermore, cellobiose and glucose were only detected at the early stage of batches, which could have been present in the pre-cultures, inoculated into the bioreactors, and were taken up in 24 hours. Cellobiose and glucose could not be detected in the course of co-cultures which indicated their immediate consumption in the co-culture. In conclusion, in this study we showed a strong synergism between the two species of clostridia in the co-culture, and found that $C$. acetobutylicum enables C. cellulolyticum to grow under harsh co-culture environment. This synergy can be attributed to the production of some growth precursors, and future metabolomic studies of this co-culture can identify such metabolites.

\section{Conclusions}

Examining of the metabolism in this clostridial co-culture along with the mono-cultures revealed that significant increase in the rate of cellulose hydrolysis can be achieved using the co-culture and making use of the synergism existing between the two clostridial species. It is likely that $C$. acetobutylicum improves the cellulolytic activity of C. cellulolyticum in the co-culture through exchange of metabolites such as pyruvate, enabling it to grow and metabolize cellulose under harsh co-culture conditions. This clostridial co-culture can offer a considerable potential CBP approach for producing commodity chemicals from cellulosic biomass, taking advantage of $C$. acetobutylicum metabolic potential in converting sugars to variety of chemicals.

\section{Methods}

\section{Strains and media}

C. acetobutylicum ATCC 824 was grown in clostridial growth medium (CGM) containing per litre: $0.75 \mathrm{~g}$ of 
$\mathrm{KH}_{2} \mathrm{PO}_{4}, 0.75 \mathrm{~g}$ of $\mathrm{K}_{2} \mathrm{HPO}_{4}, 1 \mathrm{~g}$ of $\mathrm{NaCl}, 0.01 \mathrm{~g}$ of $\mathrm{MnSO}_{4}$, $0.004 \mathrm{~g}$ of $p$-aminobenzoic acid, $0.348 \mathrm{~g}$ of $\mathrm{MgSO}_{4}, 0.01 \mathrm{~g}$ of $\mathrm{FeSO}_{4}, 2 \mathrm{~g}$ of asparagine, $5 \mathrm{~g}$ of yeast extract, $2 \mathrm{~g}$ of $\left(\mathrm{NH}_{4}\right)_{2} \mathrm{SO}_{4}$ and $50 \mathrm{~g}$ of glucose (pH 5.8). C. cellulolyticum ATCC 35317 was maintained and cultivated on a modified $\mathrm{CM} 3$ medium containing per litre: $\mathrm{KH}_{2} \mathrm{PO}_{4}, 1.5$ g; $\mathrm{K}_{2} \mathrm{HPO}_{4}$, 2.9 g; $\left(\mathrm{NH}_{4}\right)_{2} \mathrm{SO} 4,1.3$ g; $\mathrm{MgCl}_{2} .6 \mathrm{H}_{2} \mathrm{O}, 1 \mathrm{~g} ; \mathrm{CaCl}_{2}, 0.15$; $\mathrm{FeSO}_{4}, 1.25 \mathrm{mg}$; resazurin, $1 \mathrm{mg}$; cysteine hydrochloride, $1 \mathrm{~g}$; fibrous cellulose (Sigma-Aldrich, C6288), $7.5 \mathrm{~g}$, and $2 \mathrm{~g}$ of yeast extract ( $\mathrm{pH} 7.2$ ).

The co-culture medium was composed of $0.75 \mathrm{~g}$ of $\mathrm{KH}_{2} \mathrm{PO}_{4}, 1 \mathrm{~g}$ of $\mathrm{MgCl}_{2} \cdot 6 \mathrm{H}_{2} \mathrm{O}, 0.15 \mathrm{~g}$ of $\mathrm{CaCl}_{2}, 1 \mathrm{mg}$ of resazurin, $1 \mathrm{~g}$ of cysteine hydrochloride, $0.01 \mathrm{~g}$ of $\mathrm{MnSO}_{4}$, $0.004 \mathrm{~g}$ of $p$-aminobenzoic acid, $10 \mathrm{mg}$ of $\left(\mathrm{NH}_{4}\right)_{2} \mathrm{Mo}_{7} \mathrm{O}_{24}$, $0.01 \mathrm{~g}$ of $\mathrm{FeSO}_{4}, 2 \mathrm{~g}$ of asparagine, $3 \mathrm{~g}$ of yeast extract, $1 \mathrm{~g}$ of $\left(\mathrm{NH}_{4}\right)_{2} \mathrm{SO}_{4}$ and $20 \mathrm{~g}$ of fibrous cellulose per litre of medium. All pre-cultures were incubated at $37^{\circ} \mathrm{C}$ without shaking in serum bottles to reach the total protein concentration of 15 to $20 \mathrm{mg} / \mathrm{L}$ (Bio-Rad Protein Assay, 500-0006).

\section{Co-culture experiments}

All batch cultivations were conducted in $5 \mathrm{~L}$ bioreactors with a working volume of $2 \mathrm{~L}$ at $37^{\circ} \mathrm{C}$, where agitation was set at $200 \mathrm{rpm}$, and the volume of $C$. cellulolyticum inoculum at exponential growth phase formed about $10 \%$ of the total volume. The C. cellulolyticum pre-cultures were transferred twice on the co-culture medium with $7.5 \mathrm{~g} / \mathrm{l}$ of cellulose before inoculation into bioreactors, where the biomass concentration in the bioreactor inocula was between $6 \times 10^{8}$ and $8 \times 10^{8}$ cell/mL. For the co-culture batches, C. cellulolyticum was first cultivated for 48 hours at pH 7.0; then $200 \mathrm{~mL}$ of C. acetobutylicum culture at exponential phase was centrifuged and the cell pellets were suspended in $20 \mathrm{~mL}$ of CGM medium and inoculated into the bioreactor. The $\mathrm{pH}$ then was adjusted and maintained at 6.0 using $1 \mathrm{~N} \mathrm{H}_{2} \mathrm{SO}_{4}$ and $3 \mathrm{~N} \mathrm{NaOH}$. The bioreactors were kept under anaerobic conditions by continuous sparging of nitrogen gas. The same $\mathrm{pH}$ adjustment and medium were applied for the $C$. cellulolyticum mono-culture batch unless for the mono-culture batch at $\mathrm{pH} 7.2$ that was conducted on CM3 medium with no $\mathrm{pH}$ profile.

\section{Analysis}

Concentration of sugars, organic acids and alcohols were measured using Dionex Ultimate 3000 HPLC (Bio-Rad, Hercules, CA) equipped with a UV monitor $(210 \mathrm{~nm})$ and a Shodex RI-101 differential refractive index detector. The products were separated using a Bio- Rad HPX-87H cation-exchange column, where $10 \mathrm{mM} \mathrm{H}_{2} \mathrm{SO}_{4}$ was used as the mobile phase $\left(0.6 \mathrm{~mL} / \mathrm{min}\right.$ flow rate, $60^{\circ} \mathrm{C}$ column temperature, $20 \mu \mathrm{L}$ injection volume). To account for the extracellular proteins, amino acids and amino compounds in cell cultures [36], and to estimate the overall carbon recovery, total organic carbon (TOC) concentrations of the sample supernatants were quantified using Total Organic Carbon Analyzer (TOC- $\mathrm{V}_{\mathrm{CPH} / \mathrm{CPV}}$, Shimadzu, Japan), with the lower detection limit of about $0.5 \mathrm{mg} / \mathrm{L}$ using standard TOC catalyst. Cellulose concentration was measured using Updegraff method [41]. Cellulose pellets were washed using acetic acid-nitric acid reagent and water to remove the non-cellulosic material; cellulose then was quantified using anthron in a colorimetric method. The results of cellulose assay and TOC measurements showed that about $24 \%$ of the total carbon was used for $\mathrm{CO}_{2}$ formation, which was consistent with our previous model prediction [42].

To measure protein, $0.5 \mathrm{ml}$ of cell culture was centrifuged at 8,000 $\mathrm{g}$ for $2 \mathrm{~min}$ and washed with $0.9 \%$ (wt/vol) $\mathrm{NaCl}$. The pellet was resuspended in $0.5 \mathrm{ml}$ of $0.2 \mathrm{~N}$ $\mathrm{NaOH}$, and this suspension was placed in a boiling water bath for $10 \mathrm{~min}$. After cooling, the hydrolyzed sample was centrifuged as described above, and the total solubilized protein concentration in supernatants was measured using Bio-Rad Protein Assay (500-0006). Quantification of the population of each species in the co-culture was conducted using quantitative PCR (qPCR) method, which includes DNA extraction, PCR amplification of 16SrRNA gene and detection using fluorescent dyes, and is elaborated in following sections.

\section{Primer design and $q P C R$ standard plasmid DNA preparation} The qPCR primers were designed to target the 16SrRNA genes in C. acetobutylicum (CA_Cr001) and C. cellulolyticum (Ccel_R0007) as reported in Table 1. The qPCR standard plasmid solutions for each target species were prepared by cloning the purified PCR products into $\mathrm{pCR}^{\circledR} 2.1-\mathrm{TOPO}^{\odot}$ vector using $\mathrm{TOPO}^{-\mathrm{TA}}$ Cloning ${ }^{\odot}$ kit (Invitrogen $\left.^{\mathrm{Tm}}, \mathrm{K} 4500-01\right)$. In the first step, fresh PCR products were purified using GeneJET PCR Purification Kit (Fermentas, K0701,K0702); the nucleic acid concentrations in purified PCR products were measured to be 67 and 50 $\mathrm{ng} / \mu \mathrm{l}$ for $C$. acetobutylicum and C. cellulolyticum, respectively, using NanoDrop 1000 (Thermo Scientific) spectrophotometer, and PCR products purity were verified using agarose gel electrophoresis. Plasmid extraction from the positive clones was conducted using GenElute Plasmid Miniprep kit (Sigma-Aldrich, USA). All of the extracted plasmids were sequenced at the University of Toronto Sanger Sequencing Facility, and the plasmids with right inserts were selected and applied for qPCR calibrations.

There are eleven 16srRNA genes in C. acetobutylicum genome (CA_Cr001, CA_Cr004, CA_Cr007, CA_Cr010, CA_Cr013, CA_Cr016, CA_Cr019, CA_Cr022, CA_Cr025, CA_Cr028, CA_Cr033), and eight 16srRNA genes in C. cellulolyticum genome (Ccel_R0007, Ccel_R0018, Ccel_R0088, Ccel_R0084, Ccel_R0059, Ccel_R0012, Ccel_R0024, Ccel_ R0036). Multiple sequence alignment of the 16srRNA genes 
Table 1 Primer sequences used for qPCR analysis

\begin{tabular}{llll}
\hline Primer name & Target species & Primer sequence $\mathbf{( 5}^{\prime}-\mathbf{3}^{\prime}$ ) & Product length \\
\hline CA2 (forward) & C. acetobutylicum & CTTGTGGTGAGGTAACGG & $386 \mathrm{bp}$ \\
CA2 (reverse) & C. acetobutylicum & CACTCCAGACATCCAGTTG & \\
CC2 (forward) & C. cellulolyticum & TACAGGGGATAACACAGG & $348 \mathrm{bp}$ \\
CC2 (reverse) & C. cellulolyticum & CGTGGCTTATTCTCAGGTAC & \\
\hline
\end{tabular}

for each species using ClustalW2 [43] showed identical amplification regions for each set of 16SrRNA genes, therefore 8 amplicons with the same size are being produced per copy of C. cellulolyticum genome in a qPCR reaction, and 11 amplicons with the same size are being produced per copy of $C$. acetobutylicum genome in a qPCR reaction; these facts also were verified by observing single peaks in melting curve analysis as well as single DNA bands for each species in agarose gel electrophoresis runs, and must be considered in qPCR quantifications of each species. For each qPCR run a new standard curve was made using fresh dilutions, where the standard curve concentrations of $10^{8}$ copies $/ \mu \mathrm{L}$ to $10^{1}$ copies $/ \mu \mathrm{L}$ were prepared by making serial 1:10 dilutions starting with the $10^{9}$ copies/ $\mu \mathrm{L}$ plasmid solution.

\section{Genomic DNA isolation}

Initially the Mo Bio Laboratories UltraClean ${ }^{\bullet}$ Soil DNA Isolation Kit was applied to extract DNA from the samples. However, it was demonstrated that there was no correlation between culture size and the DNA yield using this kit for co-culture samples, as the same amount of DNA was extracted from $0.2,1,2$ and $5 \mathrm{~mL}$ of cultures; in fact this kit has been aimed for soil samples where the concentrations of biomass is typically low, and therefore there was a chance to miss significant amount of DNA in the co-culture samples. Consequently, DNA isolation using Norgen bacterial genomic DNA isolation kit (Norgen, \#17900) was tested, and it was found that there was a correlation between the sample size (in the range of 0.2 to $0.5 \mathrm{~mL}$ ) and DNA yield using Norgen kit, while it had a higher DNA yield compared to the Mo Bio kit. Therefore, the Norgen kit was chosen for DNA isolations; culture samples $(0.5 \mathrm{~mL})$ were centrifuged at $13000 \mathrm{~g}$ for 2 minutes, and the cell pellets were used for DNA isolations following the standard kit protocol, where $400 \mu \mathrm{L}$ elution buffer was used lastly to elute DNA in two steps ( $200 \mu \mathrm{L}$ for each dilution step).

\section{qPCR reaction preparation, detection and quantification}

The qPCR amplification was performed using $2 \mu \mathrm{L}$ of tenfold diluted sample genomic DNA and $18 \mu \mathrm{L}$ of a master mix in a total reaction volume of $20 \mu \mathrm{L}$. A master mix was prepared for each qPCR run whereby the reagents for the individual reactions were combined prior to plate preparation in order to minimize errors. Master mixes were prepared by combining $10 \mu \mathrm{L}$ of SsoFast ${ }^{\mathrm{TM}}$ EvaGreen $^{\odot}$ Supermix (Bio-Rad, \#172-5200), $1 \mu \mathrm{L}$ of each primer with final concentration of $0.25 \mu \mathrm{M}$, and $6 \mu \mathrm{L}$ of water for each reaction, and $2 \mu \mathrm{L}$ of DNA solution was added to each reaction in a $20 \mu \mathrm{L}$ reaction volume.

The qPCR amplifications and detections were carried out in a CFX96 ${ }^{\mathrm{TM}}$ Real-Time PCR Detection System (Bio-Rad Laboratories Inc.). The qPCR program had the following protocol: 1) initial denaturation at $98^{\circ} \mathrm{C}$ for $\left.3 \mathrm{~min}, 2\right) 40$ cycles of denaturation at $98^{\circ} \mathrm{C}$ for $5 \mathrm{sec}, 3$ ) annealing and extension at $60^{\circ} \mathrm{C}$ for $10 \mathrm{sec}$ followed by a plate read; afterward a melting curve analysis from 65 to $95^{\circ} \mathrm{C}$ measuring fluorescence every $0.5^{\circ} \mathrm{C}$. For all qPCR runs, the qPCR signals were analyzed at a standard pre-determined threshold of $1 \mathrm{E}+03 \mathrm{RFU}$ which was in the exponential amplification phase and above the background fluorescence noise for all the qPCR runs. The quantification cycles $\left(\mathrm{C}_{\mathrm{q}}\right.$ or $\left.\mathrm{C}_{\mathrm{T}}\right)$, the cycle number where fluorescence increases above the threshold, were used to find the DNA copy numbers (automatically calculated from the standard curve). To examine the quality of qPCR assays, standard curve with coefficient of determination $\left(\mathrm{R}^{2}\right)>0.980$, high amplification efficiency (90-110\%), and consistency across replicate reactions were considered.

\section{Additional file}

Additional file 1: Figure S1. (a) Time profiles of lactate (triangle), acetate (strike), butyrate (circle) and ethanol (rhombus), and (b) time profiles of pyruvate (triangle) and butanol (rhombus) in the co-culture experiment A. Pyruvate uptake and butanol formation were observed in the co-cultures. Figure S2. C. acetobutylicum biomass profiles in the monocultures under co-culture conditions. (a) C. acetobutylicum was cultivated on co-culture medium at $\mathrm{pH}$ of 6.0 and $20 \mathrm{~g} / \mathrm{L}$ cellulose without glucose/ pyruvate addition, and characterized using QPCR (rhombus) and hemacytometry (square). (b) C. acetobutylicum culture on co-culture medium at $\mathrm{pH}$ of 6.0 and $20 \mathrm{~g} / \mathrm{L}$ cellulose. $1 \mathrm{~g} / \mathrm{L}$ glucose was added at day 12, and 1 $\mathrm{g} / \mathrm{L}$ pyruvate was added to the culture on day 19.

\section{Abbreviations}

TOC: Total organic carbon; CBP: Consolidated bioprocessing; DS: Degree of synergism.

Competing interests

The authors declare that they do not have any competing interests.

\section{Authors' contributions}

FS conceived of the study, designed and conducted the experiments, and drafted the manuscript. RM conceived of the study and helped to draft the manuscript. Both authors read and approved the final manuscript. 


\section{Acknowledgments}

This work was supported by Natural Sciences and Engineering Research Council of Canada (NSERC) Discovery grant. Authors are grateful to Professor Edgar Acosta for providing access to Total Organic Carbon Analyzer.

Received: 18 March 2013 Accepted: 17 October 2013

Published: 4 November 2013

\section{References}

1. Lynd LR, Weimer PJ, Van ZyI WH, Pretorius IS: Microbial cellulose utilization: fundamentals and biotechnology. Microbiol Mol Biol Rev 2002, 66:506-577.

2. Lynd LR, Van Zyl WH, McBride JE, Laser M: Consolidated bioprocessing of cellulosic biomass: an update. Curr Opin Biotechnol 2005, 16:577-583.

3. Lynd LR, Currie D, Ciazza N, Herring C, Orem N: Consolidated bioprocessing of cellulosic biomass to ethanol using thermophylic bacteria. In Bioenergy, Part I Biotechnology, Volume 5. Edited by Wall JD, Harwwod CS, Demain A. Washington, DC: ASM Press; 2008:55-74.

4. Olson DG, McBride JE, Joe Shaw A, Lynd LR: Recent progress in consolidated bioprocessing. Curr Opin Biotechnol 2012, 23:396-405.

5. Hasunuma T, Okazaki F, Okai N, Hara KY, Ishii J, Kondo A: A review of enzymes and microbes for lignocellulosic biorefinery and the possibility of their application to consolidated bioprocessing technology. Bioresour Technol 2013, 135:513-522.

6. Shaw AJ, Podkaminer KK, Desai SG, Bardsley JS, Rogers SR, Thorne PG, Hogsett DA, Lynd LR: Metabolic engineering of a thermophilic bacterium to produce ethanol at high yield. Proc Natl Acad Sci U S A 2008, 105:13769-13774.

7. Argyros DA, Tripathi SA, Barrett TF, Rogers SR, Feinberg LF, Olson DG, Foden JM, Miller BB, Lynd LR, Hogsett DA, Caiazza NC: High ethanol Titers from cellulose by using metabolically engineered thermophilic, anaerobic microbes. Appl Environ Microbiol 2011, 77:8288-8294.

8. Yang SJ, Kataeva I, Hamilton-Brehm SD, Engle NL, Tschaplinski TJ, Doeppke C, Davis M, Westpheling J, Adams MW: Efficient degradation of lignocellulosic plant biomass, without pretreatment, by the thermophilic anaerobe "Anaerocellum thermophilum" DSM 6725. Appl Environ Microbiol 2009, 75:4762-4769.

9. Svetlitchnyi VA, Kensch O, Falkenhan DA, Korseska SG, Lippert N, Prinz M, Sassi J, Schickor A, Curvers S: Single-step ethanol production from lignocellulose using novel extremely thermophilic bacteria. In Biotechnology for Biofuels, Volume 6. ; 2013.

10. Li Y, Tschaplinski TJ, Engle NL, Hamilton CY, Rodriguez M, Liao JC, Schadt CW, Guss AM, Yang Y, Graham DE: Combined inactivation of the Clostridium cellulolyticum lactate and malate dehydrogenase genes substantially increases ethanol yield from cellulose and switchgrass fermentations. In Biotechnolofy for Biofuels, Volume 5; 2012

11. Higashide W, Li Y, Yang Y, Liao JC: Metabolic engineering of Clostridium cellulolyticum for production of isobutanol from cellulose. Appl Environ Microbiol 2011, 77:2727-2733

12. Hasunuma $T$, Kondo A: Development of yeast cell factories for consolidated bioprocessing of lignocellulose to bioethanol through cell surface engineering. Biotechnol Adv 2012, 30:1207-1218.

13. Yu EKC, Deschatelets $L$, Saddler JN: The bioconversion of wood hydrolyzates to butanol and butanediol. Biotechnol Lett 1984, 6:327-332.

14. Fond O, Engasser J-M, Matta-El-Amouri G, Petitdemange H: Acetone butanol fermentation on glucose and xylose. I. Regulation and kinetics in batch cultures. Biotechnol Bioeng 1986, 28:160-166.

15. Ali MK, Rudolph FB, Bennett GN: Thermostable xylanase10B from Clostridium acetobutylicum ATCC824. J Ind Microbiol Biotechnol 2004, 31:229-234.

16. Lee J, Jang YS, Choi SJ, Im JA, Song H, Cho JH, Seung DY, Terry Papoutsakis E, Bennett GN, Lee SY: Metabolic engineering of clostridium acetobutylicum ATCC 824 for isopropanol-butanol-ethanol fermentation. Appl Environ Microbiol 2012, 78:1416-1423.

17. Demain AL, Newcomb M, Wu JHD: Cellulase, clostridia, and ethanol. Microbiol Mol Biol Rev 2005, 69:124-154.

18. Jones DT, Woods DR: Acetone-butanol fermentation revisited. Microbiol Rev 1986, 50:484-524.

19. Petitdemange E, Fond O, Caillet F, Petitdemange H, Gay R: A novel one step process for cellulose fermentation using mesophilic cellulolytic and glycolytic Clostridia. Biotechnology Letters 1983, 5:119-124.
20. Mitchell WJ, Albasheri KA, Yazdanian M: Factors affecting utilization of carbohydrates by clostridia. FEMS Microbiol Rev 1995, 17:317-329.

21. Desvaux M, Guedeon E, Petitdemange H: Metabolic flux in cellulose batch and cellulosefed continuous cultures of Clostridium cellulolyticum in response to acidic environment. Microbiology 2001, 147:1461-1471.

22. Desvaux M, Guedon E, Petitdemange H: Cellulose catabolism by Clostridium cellulolyticum growing in batch culture on defined medium. Appl Environ Microbiol 2000, 66:2461-2470.

23. Lee SF, Forsberg CW, Gibbins LN: Cellulolytic activity of Clostridium acetobutylicum. Appl Environ Microbiol 1985, 50:220-228.

24. Sabathe F, Blaich A, Soucaille P: Characterization of the cellulolytic complex (cellulosome) of Clostridium acetobutylicum. FEMS Microbiol Lett 2002, 217:15-22.

25. Guedon E, Desvaux M, Payot S, Petitdemange H: Growth inhibition of Clostridium cellulolyticum by an inefficiently regulated carbon flow. Microbiology 1999, 145:1831-1838.

26. Lu Y, Zhang YHP, Lynd LR: Enzyme-microbe synergy during cellulose hydrolysis by Clostridium thermocellum. Proc Natl Acad Sci U S A 2006, 103:16165-16169.

27. Zhang YHP, Lynd LR: Toward an aggregated understanding of enzymatic hydrolysis of cellulose: noncomplexed cellulase systems. Biotechnol Bioeng 2004, 88:797-824

28. Hu J, Arantes V, Saddler JN: The enhancement of enzymatic hydrolysis of lignocellulosic substrates by the addition of accessory enzymes such as xylanase: is it an additive or synergistic effect? In Biotechnology for Biofuels, Volume 4. ; 2011

29. Russell JB, Wilson DB: Why Are Ruminal Cellulolytic Bacteria Unable to Digest Cellulose at Low pH? J Dairy Sci 1996, 79:1503-1509.

30. Flythe MD, Russell JB: Fermentation acids inhibit amino acid deamination by Clostridium sporogenes MD1 via mechanism involving a decline in intracellular glutamate rather than protonmotive force. Microbiology 2006, 152:2619-2624.

31. Roe AJ, McLaggan D, Davidson I, O'Byrne C, Booth IR: Perturbation of anion balance during inhibition of growth of Escherichia coli by weak acids. J Bacteriol 1998, 180:767-772.

32. Roe AJ, O'Byrne C, McLaggan D, Booth IR: Inhibition of Escherichia coli growth by acetic acid: A problem with methionine biosynthesis and homocysteine toxicity. Microbiology 2002, 148:2215-2222.

33. Bahl H, Gottwald M, Kuhn A, Rale V, Andersch W, Gottschalk G: Nutritional factors affecting the ratio of solvents produced by clostridiumacetobutylicum. Appl Environ Microbiol 1986, 52:169-172.

34. Desvaux M: Clostridium cellulolyticum: Model organism of mesophilic cellulolytic clostridia. FEMS Microbiol Rev 2005, 29:741-764.

35. Levin DB, Islam R, Cicek N, Sparling R: Hydrogen production by Clostridium thermocellum 27405 from cellulosic biomass substrates. Int J Hydrogen Energ 2006, 31:1496-1503.

36. Guedon E, Payot S, Desvaux M, Petitdemange $H$ : Carbon and electron flow in Clostridium cellulolyticum grown in chemostat culture on synthetic medium. J Bacteriol 1999, 181:3262-3269.

37. Guedon E, Desvaux M, Petitdemange H: Improvement of cellulolytic properties of Clostridium cellulolyticum by metabolic engineering. Appl Environ Microbiol 2002, 68:53-58.

38. Janati-Idrissi $R$, Junelles AM, El Kanouni A, Petitdemange $H$, Gay R: Pyruvate fermentation by Clostridium acetobutylicum. Biochem Cell Biol 1989, 67:735-739.

39. Jiao Y, Navid A, Stewart BJ, McKinlay JB, Thelen MP, Pett-Ridge J: Syntrophic metabolism of a co-culture containing Clostridium cellulolyticum and Rhodopseudomonas palustris for hydrogen production. International Journal of Hydrogen Energy 2012, 37:11719-11726.

40. Junelles AM, Janati-Idrissi R, Petitdemange H, Gay R: Effect of pyruvate on glucose metabolism in Clostridium acetobutylicum. Biochimie 1987, 69:1183-1190.

41. Updegraff DM: Semimicro determination of cellulose inbiological materials. Anal Biochem 1969, 32:420-424.

42. Salimi F, Zhuang K, Mahadevan R: Genome-scale metabolic modeling of a clostridial co-culture for consolidated bioprocessing. Biotechnol J 2010, 5:726-738

43. ClustalW2. http://www.ebi.ac.uk/Tools/msa/clustalw2/.

doi:10.1186/1472-6750-13-95

Cite this article as: Salimi and Mahadevan: Characterizing metabolic interactions in a clostridial co-culture for consolidated bioprocessing. BMC Biotechnology 2013 13:95. 